\title{
Gastroschisis and Omphalocele: A Case report
}

\author{
Kayastha $\mathbf{P}^{1}$, Paudel S ${ }^{1}$, Ghimire R K ${ }^{1}$, Ansari MA ${ }^{1}$ \\ ${ }^{1}$ Department of Radiology and Imaging, Institute of Medicine, Tribhuvan University \\ Teaching Hospital, Kathmandu, Nepal
}

\begin{abstract}
Fetal gastroschisis and omphalocele are congenital defects of abdominal wall that are often diagnosed by prenatal ultrasound done for routine screening or for obstetric indications such as evaluating an elevated maternal serum alpha fetoprotein (AFP). Prenatal ultrasound could potentially identify the overwhelming majority of abdominal wall defects and accurately distinguish omphalocele from gastroschisis. Here we report two cases of gastroschisis and omphalocele diagnosed at routine prenatal ultrasound.
\end{abstract}

Keywords: Gastroschisis, Omphalocele, Ultrasonography

\section{Introduction}

Omphalocele and Gastroschisis are the two most common major congenital abdominal wall defects. ${ }^{1}$ Although textbooks group the two entities together, they are separate and distinct and have many important differences in pathology and associated conditions that explain the differences in treatment plans and outcomes. Understanding the similarities and differences between gastroschisis and omphalocele is essential for patient management. Gastroschisis is a full-thickness defect in the abdominal wall usually just to the right of a normal insertion of the umbilical cord into the body wall. A variable amount of intestine and occasionally parts of other abdominal organs are herniated outside the abdominal wall with no covering membrane or sac.

Correspondence to: Dr. Prakash Kayastha Department of Radiology and Imaging, Institute of Medicine, TUTH, Kathmandu Nepal

Email: dr_prakash_kayastha@hotmail.com
An omphalocele (also known as exomphalos) is a midline abdominal wall defect of variable size, with the herniated viscera covered by a membrane consisting of peritoneum on the inner surface, amnion on the outer surface, and Wharton's jelly between the layers. The umbilical vessels insert into the membrane and not the body wall. Here we present two cases, one gastroschisis and another omphalocele which were diagnosed in routine antenatal ultrasound.

\section{Case Report}

Case 1: A 20 year old primigravida was sent for routine anomaly scan at 20 weeks of gestation. She was on regular iron and calcium and didn't have any major diseases. Calculated gestational age by ultrasonography was 19 weeks and 5 days. On ultrasonography of the abdominal region, we found defect in the anterior abdominal wall of the fetus. Bowel loops were seen herniating into the amniotic cavity and were floating without any covering membrane [Fig: 1]. Umbilical cord was seen on the left 


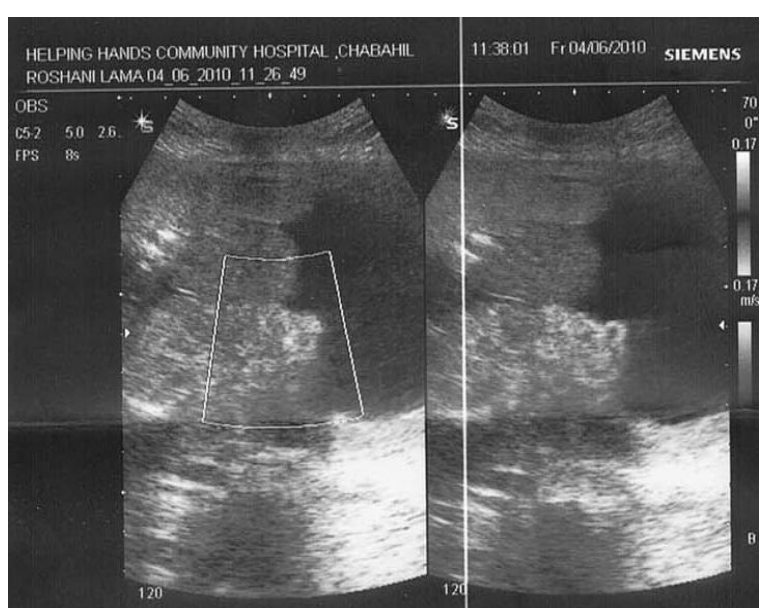

Fig 1: B mode ultrasonography image showing abdominal wall defect with herniation of bowel loops into amniotic cavity. No covering membrane is seen.

side of the abdominal wall defect (not shown in figure). Diagnosis of gastroschisis was made.

Case 2: A 25 year second gravida female was sent for routine antenatal ultrasound at 27 weeks of gestation. Her last pregnancy was normal full term hospital delivery 4 years back with delivery of normal healthy child. She didn't have any major disease and there was no family history of delivery of abnormal child. Maternal serum AFP was not tested. Her calculated gestational age by ultrasonography was approximately 26 weeks and 2 days. On scanning of abdomen, there was large anterior abdominal wall defect in the midline with herniation of liver and bowel loops through it. The contents were covered with a membrane [Fig: 2]. Umbilical cord was inserted in the center of the covering membrane. Diagnosis of omphalocele was made.

There were no other associated anomalies in both of the fetuses. Amniotic fluid volume was normal in both cases. The findings were confirmed in both cases by three other radiologists with 3 to 15 years of experience in obstetric ultrasound. Upon counseling, both women and their family opted for termination of pregnancy rather than continuing further. They also denied for

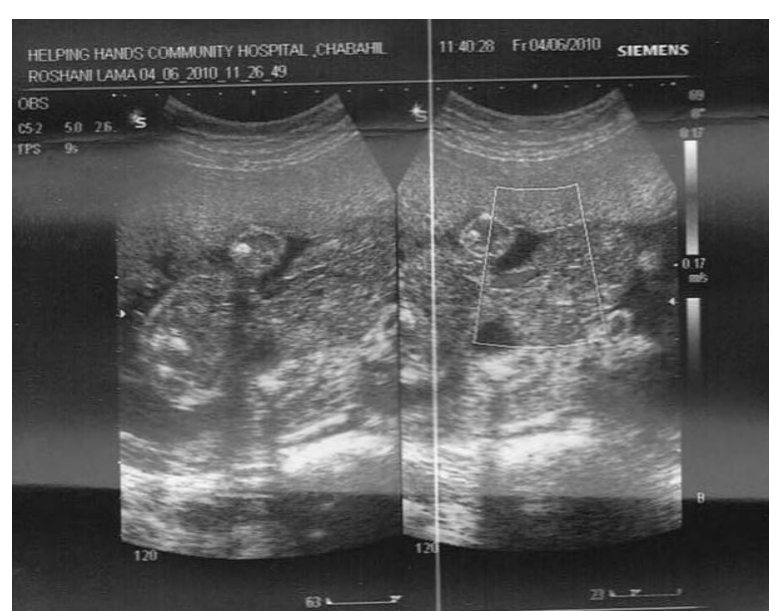

Fig 2: B mode ultrasonography image showing abdominal wall defect with herniation of liver and bowel loops into amniotic cavity. The contents are covered with a membrane.

karyotyping as it is not easily available in our country. Pregnancy was terminated and the findings were confirmed post delivery [Fig: $3 \&$ 4]. No other associated structural defects were detected on examination of the terminated fetuses. However, specimen was not sent for karyotyping or autopsy.

\section{Discussion}

Omphalocele and gastroschisis are the two most common major congenital abdominal wall defects. ${ }^{1}$ Omphalocele is a midline anterior abdominal wall defect with extrusion of abdominal viscera, covered by a membranous sac, into the base of the umbilical cord. Gastroschisis is usually a small defect in the anterior abdominal wall typically located to the right of the umbilical ring and resulting in the herniation of the abdominal contents, without a surrounding membrane, into the amniotic cavity. Both malformations are now frequently diagnosed prenatally using ultrasound scanning.

There are regional differences in the incidence of abdominal wall defects however, a rough estimate is that worldwide, the incidence of gastroschisis ranges between 0.4 and 3 per 10,000 births and seems to be increasing, whereas the incidence of omphalocele ranges between 1.5 and 3 per 


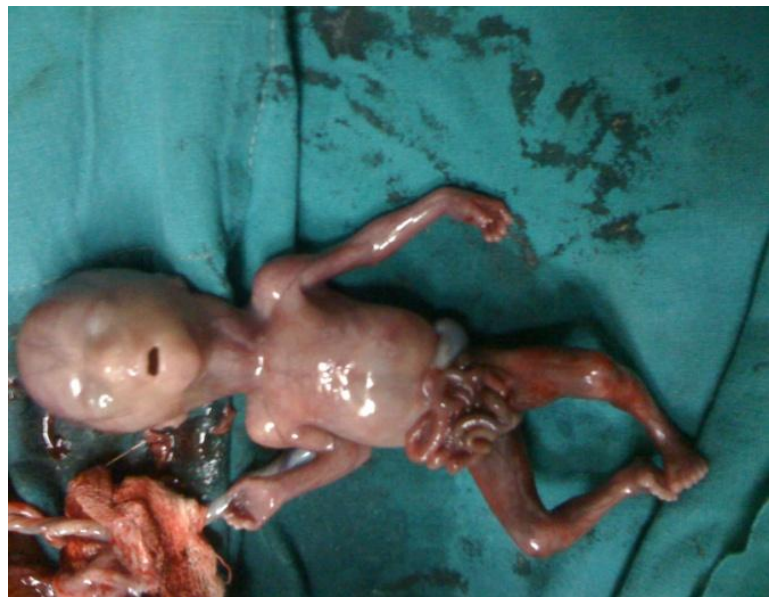

Fig 3: Post delivery photograph showing abdominal wall defect with herniation of bowel loops and no covering membrane.

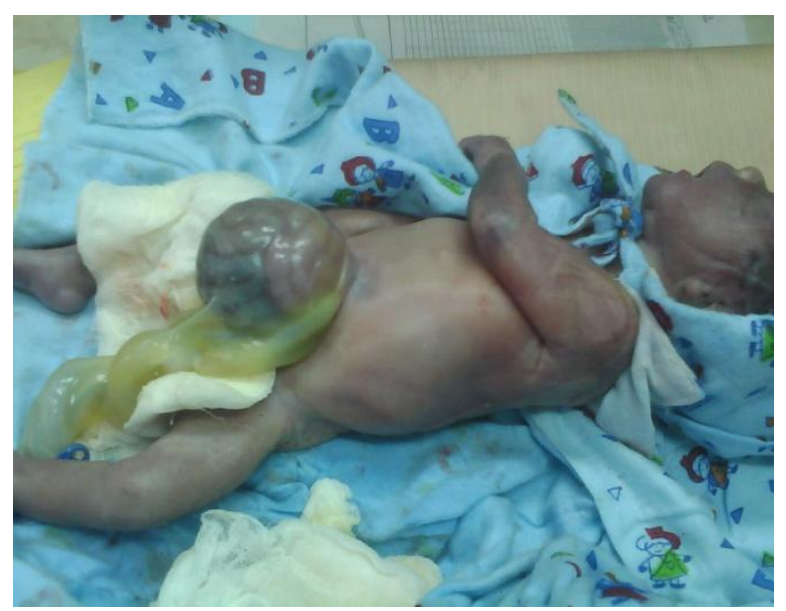

Fig 4: Post delivery photograph showing abdominal wall defect with herniation of bowel loops and liver covered with a membrane. Umbilical cord is seen inserting into the center of the covering membrane.

\section{0,000 births and is stable. ${ }^{2-4}$}

In gastroschisis, the incidence of associated anomalies is between $10 \%$ and $20 \%$, and most of the significant anomalies are in the gastrointestinal tract. ${ }^{5}$ About $10 \%$ of babies who have gastroschisis have intestinal stenosis or atresia that results from vascular insufficiency to the bowel at the time of gastroschisis development or, more commonly, from later volvulus or compression of the mesenteric vascular pedicle by a narrowing abdominal wall ring. ${ }^{6}$ Serious associated anomalies outside the abdomen or gastrointestinal tract, such as chromosomal abnormalities, are unusual.

In contrast to gastroschisis, patients with omphalocele have a very high (up to $50 \%-$ $70 \%$ ) incidence of associated anomalies. The incidence of associated anomalies is lower in liveborn patients because those who have multiple and serious anomalies are more likely to be stillborn. ${ }^{7}$ Chromosome anomalies, notably trisomy $13,14,15,18$, and 21 , are present in up to $30 \%$ of cases. Cardiac defects are also common, being present in $30 \%$ to $50 \%$ of cases. Multiple anomalies are frequent and may be clustered in syndromic patterns. One important pattern is the Beckwith-Wiedemann syndrome that may be present in up to $10 \%$ of cases. ${ }^{8}$ It may be also associated with pentalogy of Cantrell, and omphalocele exstrophy imperforate anus spinal defects syndrome.

The exact mechanism leading to omphalocele is controversial. It has been suggested that failure of the reduction of physiologic embryonic umbilical hernia results in omphalocele. Another possibility is that omphalocele results from the failure of the embryonic lateral folds to fuse in the midline.

Gastroschisis is thought to result from an ischemic insult to the developing body wall. The right paraumbilical area is an area at risk because it is supplied by the right umbilical vein and right omphalomesenteric artery until they involute. If this ordered development and involution is disturbed in degree or timing, then a body wall defect could result from the resulting body wall ischemia. An alternative hypothesis that may account for some cases of gastroschisis is that the defect results from an early rupture of a hernia of the umbilical cord.

Prenatal ultrasound could potentially identify the overwhelming majority of abdominal wall defects and accurately distinguish omphalocele from gastroschisis. This identification would permit an opportunity to counsel the family and to prepare for optimal postnatal care. It is unfortunate, however, that 
the accuracy of prenatal ultrasound for diagnosing abdominal wall defects is affected by the timing and goals of the study, fetal position, and the experience and expertise of the operator. The specificity is high (more than $95 \%$ ), but the sensitivity is only $60 \%$ to $75 \%$ for identifying gastroschisis and omphalocele. ${ }^{9,10}$

The outcome of patients who have gastroschisis depends largely on the condition of the vulnerable bowel, whereas the outcome of patients who have omphalocele depends largely on the associated anomalies and medical conditions. Overall, patients who have gastroschisis have an excellent prognosis.

\section{Conclusion}

Prenatal ultrasound could potentially identify majority of abdominal wall defects and accurately distinguish omphalocele from gastroschisis. This identification would permit an opportunity to counsel the family and to prepare for optimal postnatal care.

\section{References}

1. Stoll C, Alembik Y, Dott B, Roth MP. Risk factors in congenital abdominal wall defect (omphalocele and gastroschisis): a study in a series of 265,858 consecutive births. Ann Genet 2001;44:201-208.

2. Curry JI, McKinney P, Thornton JG, et al. The aetiology of gastroschisis. Br $\mathbf{J}$ Obstet Gynaecol 2000;107(11):1339-46.

3. Tan KH, Kilby MD, Whittle MJ, et al. Congenital anterior abdominal wall defects in England and Wales 1987-93: retrospective analysis of OPCS data. BMJ 1996;313(7062):903-6.

4. Rankin J, Dillon E, Wright C. Congenital anterior abdominal wall defects in the north of England, 19861996: occurrence and outcome. Prenat Diagn 1999;19(7):662-8.

5. Molik KA, Gingalewski CA, West KW, et al. Gastroschisis: a plea for risk categorization. J Pediatr Surg 2001;36(1):51-5.

6. Snyder CL, Miller KA, Sharp RJ, et al. Management of intestinal atresia in patients with gastroschisis. J Pediatr Surg 2001;36(10):1542-5.

7. Hwang PJ, Kousseff BG. Omphalocele and gastroschisis: an 18-year review study. Genet Med 2004;6(4):232-6.

8. Nicolaides KH, Snijders RJ, Cheng HH, et al. Fetal gastro-intestinal and abdominal wall defects:associated malformations and chromosomal abnormalities. Fetal Diagn Ther 1992;7(2):102-15

9. Rankin J, Dillon E, Wright C. Congenital anterior abdominal wall defects in the north of England, 19861996: occurrence and outcome. Prenat Diagn 1999;19(7):662-8.

10. Walkinshaw SA, Renwick M, Hebisch $\mathrm{G}$, et al. How good is ultrasound in the detection and evaluation of anterior abdominal wall defects?Br J Radiol 1992;65(772):298-301. 